\title{
Breaking the Intersubband Selection Rules for Absorption with ZnO Quantum Wells: Light Polarization Sensitivity under Normal Incidence
}

\author{
M. Montes Bajo, ${ }^{1}$ J. Tamayo-Arriola, ${ }^{1}$ N. Le Biavan, ${ }^{2}$ J. M. Ulloa, ${ }^{1}$ P. Vennéguès, ${ }^{2}$ D. Lefebvre, ${ }^{2}$ \\ M. Hugues, ${ }^{2}$ J.-M. Chauveau, ${ }^{2}$ and A. Hierro ${ }^{1, *}$ \\ ${ }^{1}$ ISOM, Universidad Politécnica de Madrid, 28040 Madrid, Spain \\ ${ }^{2}$ Université Côte d'Azur, CNRS, CRHEA, 06560 Valbonne, France
}

(Received 8 May 2018; revised manuscript received 9 July 2018; published 12 September 2018)

\begin{abstract}
Intersubband (ISB) absorption in quantum wells (QW) at normal incidence is forbidden by the selection rules for light polarization. To be useful under this convenient geometry, QW structures require postgrowth texturing of the surfaces. Here, we demonstrate polarization-sensitive ISB absorption of light at normal incidence using as-grown, nonpolar $m$-plane $\mathrm{ZnO} /(\mathrm{Mg}, \mathrm{Zn}) \mathrm{O}$ multiple $\mathrm{QWs}$ without any postprocessing. This breakage of the selection rules is possible due to the formation of a self-assembled $\mathrm{V}$-groove grating QW profile in the direction perpendicular to the $c$ axis, which lies in the growth plane. A geometrical model is developed that predicts the dependence of the ISB absorption strength with the angle of incidence and the QW V-groove angle and is verified by high-resolution transmission electron microscope analysis. As a result of the in-plane anisotropy of the QW grating geometry, a strong light polarization sensitivity is demonstrated under normal incidence, with a predicted polarization sensitivity contrast above 80:1 for a $\pm 5^{\circ}$ cone of angles.
\end{abstract}

DOI: 10.1103/PhysRevApplied.10.034022

\section{INTRODUCTION}

Intersubband (ISB) transitions between confined energy levels in a quantum well (QW) are of great interest for their use in fundamental studies of strong light-matter coupling physics [1] and nonlinear frequency conversion [2], among other topics. They are also of crucial technological interest since they are the driving physical mechanism for absorption in both quantum-well infrared photodetectors (QWIPs) and quantum cascade detectors (QCDs) operating from the near IR to the THz region $[3,4]$. The selection rules for ISB transitions for semiconductors where there is negligible mixing between valence and conduction band states require the electric field of the light to be perpendicular to the QW planes, which implies that ISB absorption under normal incidence is forbidden [3]. The nature of these transitions has thus traditionally posed challenges to the development of IR detectors for various applications, such as focal-plane arrays of detectors, where the individual detectors are oriented in such a way that the

*adrian.hierro@upm.es

Published by the American Physical Society under the terms of the Creative Commons Attribution 4.0 International license. Further distribution of this work must maintain attribution to the author(s) and the published article's title, journal citation, and DOI. light reaches the QWs at normal incidence. To circumvent this issue, postgrowth processing needs to be employed. Typically, the detectors are oriented so the light enters the device through the substrate. Under this configuration, the top layer of the structure (i.e., the last one to be reached by the incoming light) is textured as a grating so the light is diffracted and crosses the QW structure again, this time at a non-normal angle of incidence where the ISB transitions are allowed [3].

Having detectors in the IR and $\mathrm{THz}$ which are not only intensity sensitive but also polarization sensitive is even more desirable, since it will allow us to achieve information about the vector nature of the detected optical field through IR and $\mathrm{THz}$ polarimetry [5]. In the GaAs and InP systems, the sensitivity to light polarization of the devices is also achieved during the fabrication process, e.g., by etching parallel stripes on the top surface of the semiconductor layer stack [6,7], which can go as deep as to make stripes of the QWs themselves (the so-called corrugated QW approach [8]), or by the deposition of metallic gratings consisting of parallel stripes [9]. The geometry of the stripes serves to control the direction at which the light is diffracted, therefore allowing the absorption of light only when its initial polarization is perpendicular to the stripes. Also, normal-incidence ISB absorption can be achieved by the deposition of plasmonic patterned structures [2].

In order to overcome these inherent limitations to ISB transitions and to achieve polarization-sensitive ISB 
absorption at normal incidence without the need to use additional processing steps or external optical components, a metamaterial is needed that meets the following requirements: (1) high-quality-crystal QWs need to be grown in a highly controlled and reproducible fashion in order to obtain ISB transitions; (2) the active region must show a spontaneously generated morphology that facilitates the absorption of light under normal incidence; and (3) this morphology must be anisotropic, i.e., must appear only in one in-plane direction, but not in the perpendicular one, such that light polarization sensitivity can be achieved. Here, we demonstrate that these conditions are nicely met by the $\mathrm{ZnO} /(\mathrm{Mg}, \mathrm{Zn}) \mathrm{O}$ alloy family: ISB transitions from multiple quantum wells (MQWs) have already been demonstrated with excellent characteristics [10-13] and the anisotropy of its wurztize crystal structure can be exploited by growing the QWs along the $m$ axis with the $c$ axis in-plane, i.e., by using a nonpolar orientation $[10,13]$. Moreover, the nonpolar orientation facilitates the ISB transitions owing to the absence of internal electric fields that would have an impact on the oscillator strengths via the quantum-confined Stark effect. Also, the growth over readily available native substrates helps reduce the density of dislocations and other detrimental defects on the MQWs [10,13].

As reported elsewhere [14], $\mathrm{ZnO} /(\mathrm{Mg}, \mathrm{Zn}) \mathrm{O}$ QWs grown on the nonpolar $m$-plane orientation feature a $\mathrm{V}$-groove grating shape perpendicular to the $c$ axis and along the $a$ axis (both of which are contained in the plane of the sample) which appears spontaneously during molecular-beam-epitaxy (MBE) growth under a particular set of growth conditions. Figure 1(a) shows a cross-sectional high-resolution transmission-electronmicroscope (HRTEM) image of these QWs through a plane perpendicular to the $c$ axis, where the $\mathrm{V}$-groove grating pattern is apparent. In MQW structures featuring this geometry and under normal incidence of the incoming light, there are two possible scenarios for the orientation of the electric field [Fig. 1(b)]. First, if $\mathbf{E} \| c$, the electric field of the light is also parallel to the plane of the QWs everywhere in the structure. Therefore, in this configuration, there is no component of the electric field perpendicular to the plane of the MQWs and the ISB transitions are forbidden by the selection rules. This case is equivalent to that of a usual, flat MQW structure. On the contrary, if $\mathbf{E} \perp c$, there is a component of the electric field which is perpendicular to the MQWs and ISB transitions should therefore become allowed. Here, we show how a stack of these Vgroove QW gratings acts as a self-assembled metamaterial allowing for ISB absorption at normal incidence, which is forbidden in the usual, flat QW structures. Moreover, absorption of light by this metamaterial is also sensitive to the polarization of the light with respect to the $c$ axis of the crystal without the need for any postgrowth fabrication steps.

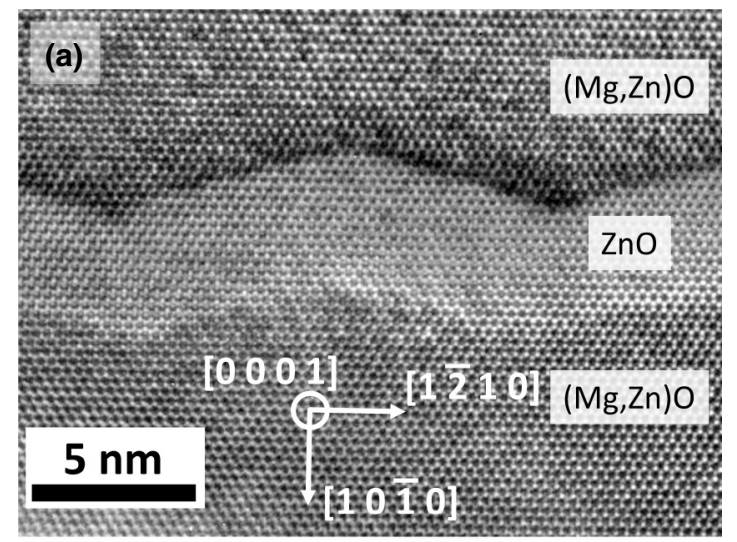

(b)

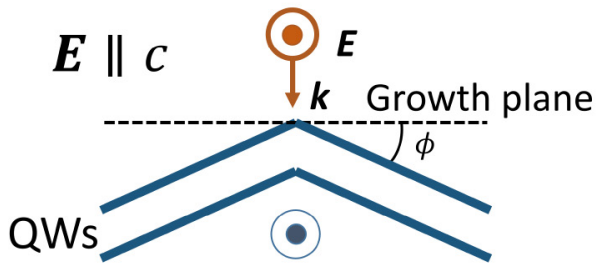

$c$ axis

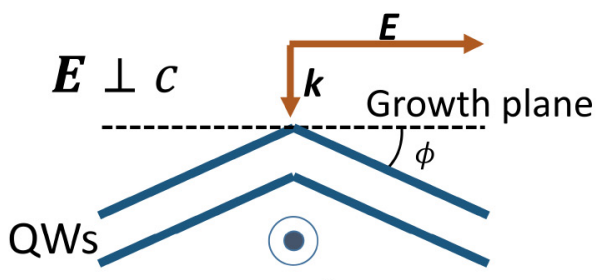

$c$ axis

FIG. 1. (a) Cross-sectional HRTEM image of sample B. (b) Schematic describing the proposed mechanism by which polarization-sensitive, normal-incidence ISB absorption is allowed in $m$-plane $\mathrm{ZnO} /(\mathrm{Mg}, \mathrm{Zn}) \mathrm{O}$ QWs. $\phi$ is the angle between the QW interface and the growth plane.

\section{EXPERIMENTAL DETAILS}

In this paper, we show the results from three $m$ plane $\mathrm{ZnO} /(\mathrm{Mg}, \mathrm{Zn}) \mathrm{O} \mathrm{MQW}$ structures grown by $\mathrm{MBE}$ on native $m$-plane $\mathrm{ZnO}$ substrates (more details are given in Ref. [13]). Figure 2(a) shows a schematic and summary of the samples. Sample A features a 15-period $\mathrm{ZnO}(3.6 \mathrm{~nm}) / \mathrm{Mg}_{0.26} \mathrm{Zn}_{0.74} \mathrm{O}(10 \mathrm{~nm}) \quad \mathrm{MQW}$ structure. Samples B and C are nominally identical except for the doping level and consist of a 10-period $\mathrm{ZnO}(3.9 \mathrm{~nm}) / \mathrm{Mg}_{0.32} \mathrm{Zn}_{0.68} \mathrm{O}(10 \mathrm{~nm}) \mathrm{MQW}$. The QWs are doped with $\mathrm{Ga}$ at three different levels. A high doping concentration is chosen in order to maximize the ISB absorption, which scales with the electron concentration in the QW [10]. The actual electron concentration in the QWs is extracted from fits to reflectance spectra taken under $p$ and $s$ polarization of the incident light at a $45^{\circ}$ angle of incidence measured in a Fourier-transform IR spectrometer 
(a)

$\times N\left\{\begin{array}{|c|l|l|l|l|l|}\hline \text { MgZnO } & L_{b} & & \text { A } & \text { B } & \text { C } \\ \hline \text { ZnO QW } & L_{Q W} & N & 15 & 10 & 10 \\ \hline \text { MgZnO } & \text { Buffer } & L_{Q W}(\mathrm{~nm}) & 3.6 & 3.9 & 3.9 \\ \hline \begin{array}{c}\text { ZnO } \\ \text { Buffer }\end{array} & 100 \mathrm{~nm} & L_{b}(\mathrm{~nm}) & 10 & 10 & 10 \\ \hline \begin{array}{c}\text { m-ZnO } \\ \text { Substrate }\end{array} & & \text { Buffer }(\mathrm{nm}) & 50 & 100 & 100 \\ \hline\end{array}\right.$

(b)

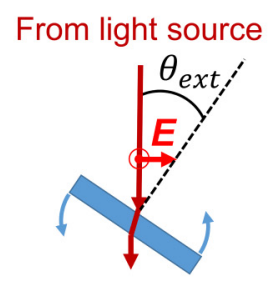

To detector

(c)

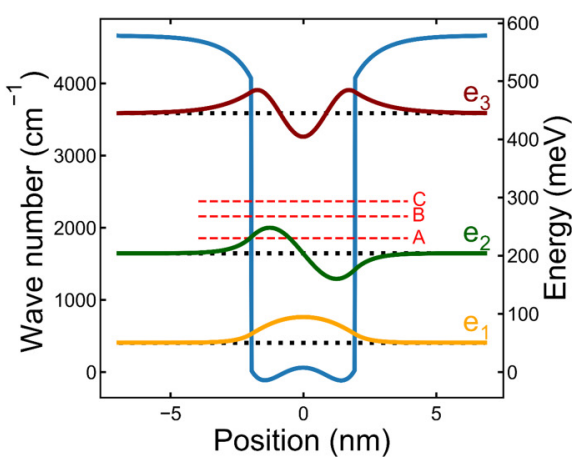

FIG. 2. (a) Schematic of the samples shown in this work. The table summarizes the relevant parameters. The electron concentration $n$ is extracted from reflectance spectroscopy as described in the text. (b) Schematic describing the experimental setup for transmittance experiments. The plane of incidence of the light is the plane of the page. The angle of incidence of the light on the sample $\theta_{\text {ext }}$ can be changed by rotating the sample around an axis perpendicular to the plane of the incidence. Both the polarization of the light and the $c$ axis of the crystal can be independently set perpendicular to or included in the plane of incidence. (c) Calculated potential profile of sample B at room temperature. All the samples presented here show calculated profiles similar to this one. The image includes the Fermi energy for all three samples (dashed red lines).

(FTIR), following the procedure described elsewhere [10]. The electron concentration in the QWs is thus found to be $6.5 \times 10^{19}, 8.5 \times 10^{19}$, and $1.05 \times 10^{20} \mathrm{~cm}^{-3}\left(2.3 \times 10^{13}\right.$, $3.1 \times 10^{13}$, and $4.1 \times 10^{13} \mathrm{~cm}^{-2}$ ) for samples A, B, and $\mathrm{C}$, respectively. The back side of the $\mathrm{ZnO}$ substrate is polished with diamond compound down to a $250-\mathrm{nm}$ grain size to facilitate the transmission measurements, which are performed in the FTIR with the sample mounted vertically on a rotating holder that allows us to change the angle of incidence of the IR light [Fig. 2(b)].

The samples are measured in high-resolution electrontransmission microscopy and selective area diffraction in ten different areas from the same TEM lamella approximately $2 \mathrm{~mm}$ in size. The angle $\varphi$ the QWs form with respect to the growth plane [Fig. 1(b)] can be deduced from the Fourier transform of the HRTEM images. In addition to the diffraction spots due to the hexagonal lattice of the $\mathrm{ZnO}$ and $(\mathrm{Mg}, \mathrm{Zn}) \mathrm{O}$ crystals, other diffraction spots are observed. By selecting only these spots and doing the inverse Fourier transform, the $\mathrm{V}$-groove profile can be extracted and enhanced compared to the initial HRTEM image. This is a proof that the additional diffraction spots are linked to the periodicity of the $\mathrm{V}$-groove profile. The angle $\phi$ is deduced from the inclination of these spots with respect to the growth direction in the reciprocal space $m^{*}$. The error on the angle is estimated from the width of the diffraction spots.

Calculation of the potential profile of the QWs by selfconsistently solving the Schrödinger and Poisson equations considering also exchange correlation effects yields a QW potential profile with three confined electronic levels [Fig. 2(c)] and a Fermi energy found between the second and third confined levels. Under these conditions, there are two allowed transitions with comparable absorption probability $\omega_{12}$ (from 1240 to $1360 \mathrm{~cm}^{-1}$ depending on the sample) and $\omega_{23}\left(1890\right.$ to $\left.1930 \mathrm{~cm}^{-1}\right)$ that have to be accounted for. As a result of dipole-dipole interaction, these two ISB transitions couple into a single one, a multisubband plasmon (MSP) [15]. The MSP resonances are calculated to be observed at 3068,3616 , and $4028 \mathrm{~cm}^{-1}$ for samples A, B, and C, respectively. Note that these MSP resonances are found at much larger energies than $\omega_{12}$ and $\omega_{23}$ as a result of the depolarization shift by which the oscillating electrons screen the electric field, producing an increase of the final MSP resonance that scales with the square root of the QW doping [16]. Details on these calculations in samples similar to those presented here are given elsewhere [10].

\section{RESULTS AND DISCUSSION}

Figure 3(a) shows the ratio of the $\mathbf{E} \| c$ to $\mathbf{E} \perp c$ transmission spectra at normal incidence for samples A, B, and $\mathrm{C}$. All the spectra show an absorption band between 2000 and $4500 \mathrm{~cm}^{-1}$, consistent with what is expected with the polarization of the light with respect to the $c$ axis [Fig. 1(b)]. To further verify that the observed peak at normal incidence is the ISB transition, the $s$-to- $p$ polarization transmittance spectra ratio measured at a $50^{\circ}$ angle of incidence is plotted in Fig. 3(b). An ISB transition peak is clearly visible, corresponding to the peaks observed at normal incidence and also consistent with the calculations of the ISB transition frequency for the three samples (see Sec. II). This confirms that what is observed at normal incidence is indeed the ISB transition. 


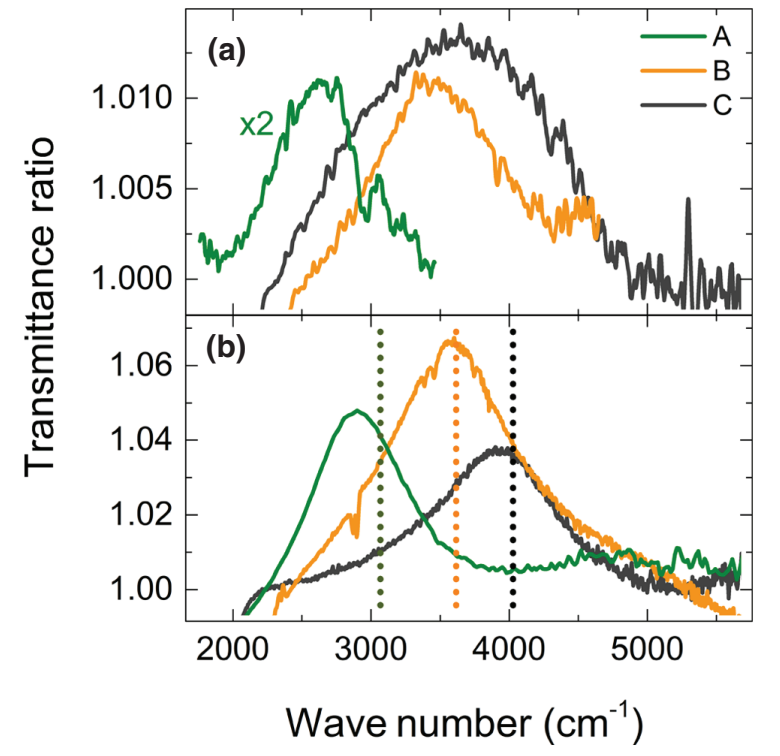

FIG. 3. Ratio of $\mathbf{E} \| c$ to $\mathbf{E} \perp c$ transmittance spectra at normal incidence (a), and ratio of $s$-to- $p$ polarization transmittance spectra at a $50^{\circ}$ angle of incidence (b), for the three samples. The dotted lines in (b) indicate the calculated MSP resonance wave number.

The relation of the observed ISB transition at normal incidence with the actual shape of the $\mathrm{V}$-groove QW grating remains to be verified. To do so, the experimental results are compared to a geometrical model that calculates the relative intensity of the ISB transition as a function of the $\mathrm{V}$-groove QW angle measured with respect to the growth plane $\phi$ and the internal angle of incidence of the light $\theta$ (Fig. 4). Note that $\theta$ is the angle of incidence with respect to the growth direction inside the QW; i.e., it is the angle of incidence after refraction at the sample surface has taken place. An isotropic index of refraction $n=1.87$ has been used for the refraction at the topmost air/(Mg,Zn)O interface of our samples and $\theta=\arcsin \left(\theta_{\text {ext }} / n_{(\mathrm{Mg}, \mathrm{Zn}) \mathrm{O}}\right)$, where $\theta_{\text {ext }}$ is the angle of incidence from air onto the sample surface. The refraction at the QW/barrier interfaces has been neglected for the sake of simplicity.

The ISB absorption coefficient in a standard, flat MQW structure depends on the angle of incidence as $\sin ^{2} \theta / \cos \theta$ [3]. The $\sin ^{2} \theta$ term is an expression of the selection rule for polarization; i.e., it comes from the quantification of the component of the electric field of the incoming light that is perpendicular to the QWs. The $\cos \theta$ term accounts for the variation of the optical path of the light across the MQW structure with the angle of incidence.

There are two geometries to be considered here. First, when the $c$ axis is perpendicular to the plane of incidence of the $p$-polarized light, $\mathbf{E}$ is always perpendicular to the (a)

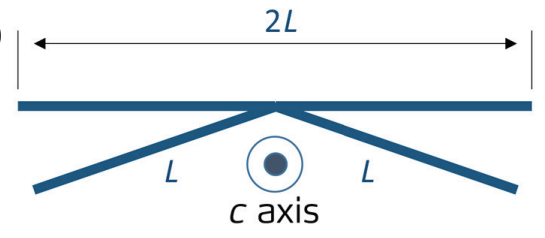

(b)

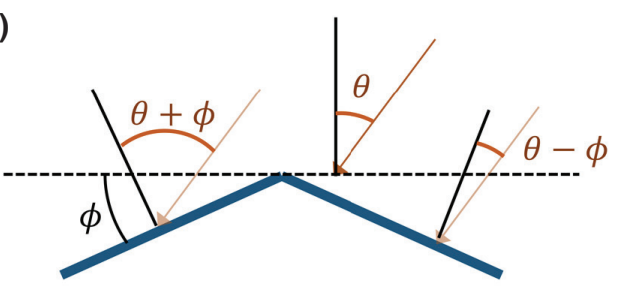

(c)

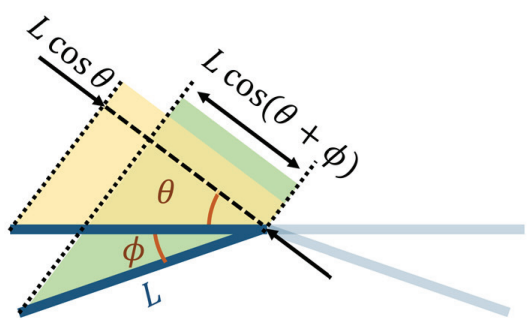

(d)

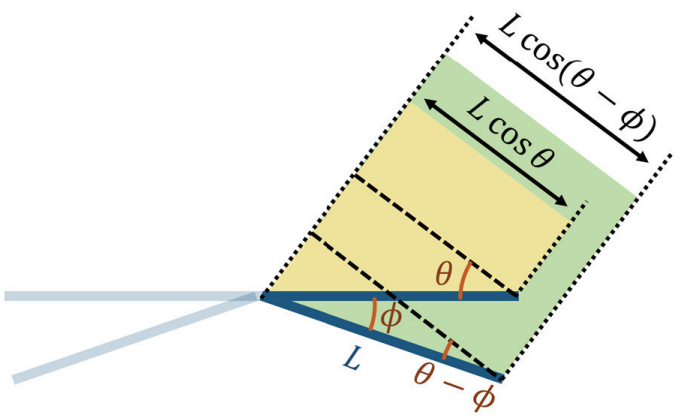

FIG. 4. Schematics describing the geometrical model. See details in text. (a) Illustration of the splitting of the problem into two halves. (b) Definition of the symbols and the effective angle of incidence. (c) Definition of the cross-sectional area as seen by the incoming light for $\phi=0$ and $\phi \neq 0$ on the left-hand side of the structure. (d) Same as (c) for the right-hand side.

$c$ axis. Second, when the $c$ axis lies in the plane of incidence of the $p$-polarized light, $\mathbf{E}$ is at an arbitrary angle with respect to the $c$ axis as $\theta$ changes. In this case, $\mathbf{E} \| c$ when $\theta=0^{\circ}$.

If we focus first on the case where $c$ is perpendicular to the plane of incidence when $\theta_{\text {ext }}=\theta=0^{\circ}$, we can consider a length $2 L$ of QW along the $a$ axis (i.e., perpendicular to the $c$ axis) when $\phi=0$ [Fig. 4(a)]. As $\phi$ increases, the problem can be divided in two halves: on the one hand, all the QW sections that are tilted clockwise and, on the other hand, all the sections that are tilted anticlockwise. In the former case, the effective angle of incidence is $\theta-\phi$ and, in the latter, it is $\theta+\phi$ [Fig. 4(b)], which yields 
an ISB absorption coefficient:

$$
\alpha_{\mathrm{ISB}} \propto\left(\frac{\sin ^{2}(\theta+\phi)}{\cos (\theta+\phi)}+\frac{\sin ^{2}(\theta-\phi)}{\cos (\theta-\phi)}\right) .
$$

It is also necessary to consider, as illustrated in Figs. 4(c) and $4(\mathrm{~d})$, that the effective cross section of each of the two halves of the model, as seen by the light incoming at a given angle of incidence, varies with $\phi$ and $\theta$. Using the notation of Fig. 4, the effective cross section for a finite $\phi$ varies with respect to the case $\phi=0$ by a factor

$$
\frac{L \cos (\theta \pm \phi)}{L \cos \theta},
$$

where the plus (minus) sign applies to the counterclockwise(clockwise-)tilted half of the QW. Therefore, we arrive at an expression for the ISB absorption coefficient given by

$$
\alpha_{\mathrm{ISB}}=A \frac{1}{\cos \theta}\left[\sin ^{2}(\theta+\phi)+\sin ^{2}(\theta-\phi)\right] .
$$

Here, $A$ is a proportionality constant that includes all of the factors in $\alpha_{\text {ISB }}$ that do not depend on $\theta$ or $\phi$. Equation (3) reduces to the expression for flat QWs when $\phi=0^{\circ}$.

When the orientation of the sample is such that the $c$ axis is contained in the plane of incidence, a similar calculation as carried out previously can be made. An $\alpha_{\mathrm{ISB}}$ intensity similar to Eq. (3) with $\phi=0^{\circ}$ is found, but corrected by an extra multiplicative $\cos \phi$ factor. As shown subsequently, $\phi$ is small in our QWs, $\cos \phi \approx 1$, and, therefore, this factor is neglected for the sake of simplicity.

To compare the results of the calculation with the experiment, the transmittance of sample B is measured as a function of the angle of incidence under $p$ polarization for both the configurations in which the $c$ axis is contained in or perpendicular to the plane of incidence (these two configurations correspond to $\mathbf{E} \| c$ and $\mathbf{E} \perp c$ when $\theta=0$, respectively, see Sec. II and Fig. 2). The integrated area of the ISB transition is evaluated as the area between the dip on the transmittance spectrum corresponding to the ISB transition and a straight baseline from 2800 to $4200 \mathrm{~cm}^{-1}$ (i.e., spanning the whole width of the ISB transition) for every spectrum [Fig. 5(a)]. Figure 5(b) shows the extracted integrated areas as a function of the angle of incidence. From the experiments performed under $p$ polarization of the incoming light and keeping the $c$ axis in the plane of incidence of the light (such that $\mathbf{E} \| c$ at normal incidence, i.e., when $\theta=0$ ), it is observed that, at normal incidence, the ISB transition intensity is not detectable, as expected (see Fig. 1). When the angle of incidence is increased, an ISB transition starts to be observed, whose intensity also increases with $\theta$. When the $c$ axis is kept perpendicular to the plane of incidence under $p$ polarization (i.e., $\mathbf{E} \perp c$ throughout the experiment), it is also observed that the ISB
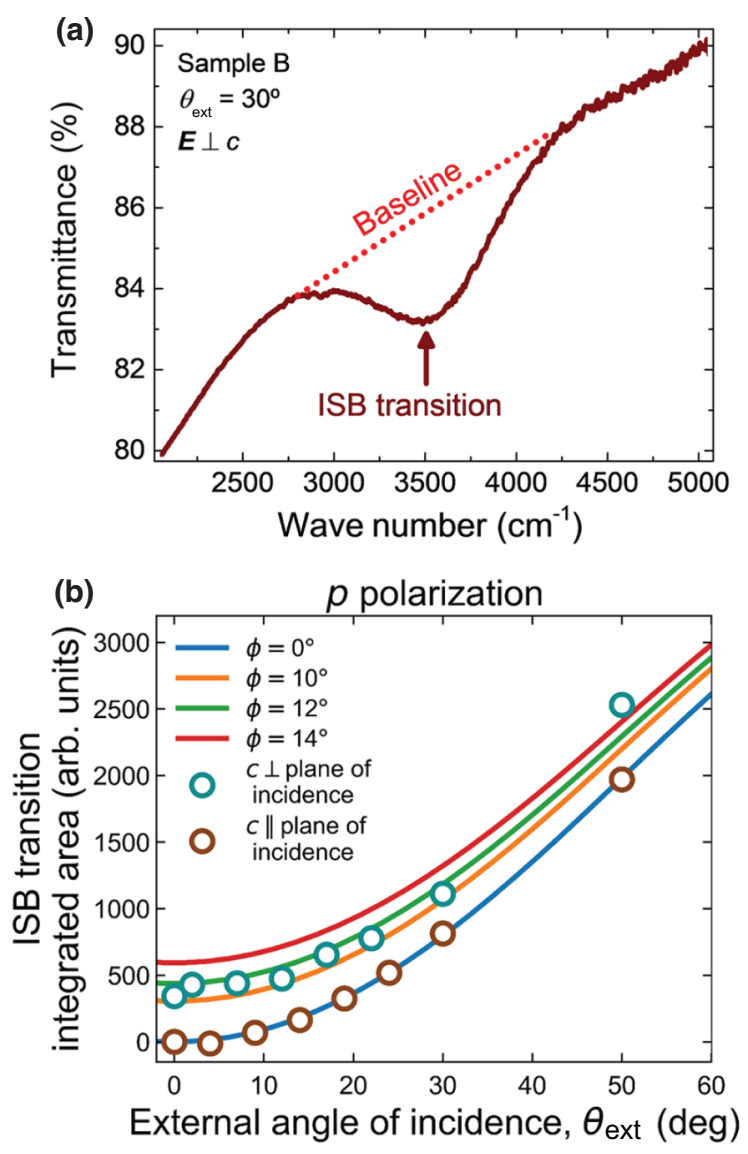

FIG. 5. (a) Illustration of the determination of the ISB transition integrated area. (b) The data points indicate the experimental ISB transition integrated area, as measured under $p$ polarization of the incoming light, as a function of the external angle of incidence when $c$ is perpendicular to the plane of incidence, so $\mathbf{E} \perp c$ for all angles (blue), and when $c$ is parallel to the plane of incidence, so $\mathbf{E} \| c$ when $\theta_{\text {ext }}=0^{\circ}$ (brown). The lines indicate the calculated ISB transition intensity from the model, adjusting $A$ so the calculation for $\phi=0$ matches the results when $c$ is parallel to the plane of incidence.

intensity increases with the increasing angle of incidence, but in this case, there is a noticeable ISB absorption even at normal incidence.

The experimental results are now compared to the calculations from the geometrical model. First, the curve corresponding to $\phi=0$ is plotted and the proportionality constant in Eq. (3) is adjusted until we get a good fit to the experimental data when the $c$ axis is contained in the plane of incidence. With the value of $A$ thus found, the curves corresponding to a series of values of $\phi$ are plotted. It is found that the experimental data obtained when $\mathbf{E} \perp c$ are delimited by the lines corresponding to $12^{\circ}<\phi<14^{\circ}$ [Fig. 5(b)]. Note that, for $\phi$ in this range, $\cos \phi \approx 1$ to within $3 \%$. Therefore, the approximation made previously of using Eq. (3) with $\phi=0^{\circ}$ when $c$ is parallel to the plane of incidence is well justified. 
The results of the estimation of $\phi$ by IR spectroscopy are now compared to what can be measured directly from the HRTEM analysis of sample B [Fig. 1(a)]. The angle between the QW edges and the growth direction can be analyzed by taking the Fourier transform of the image (see Sec. II). This analysis results in a value of $\phi=14 \pm$ $3^{\circ}$, in remarkable agreement with what is obtained from modeling the IR transmission measurements. From this agreement, the validity of the geometrical model for the estimation of $\phi$ is verified and, also, it is demonstrated that the ISB transition observed at normal incidence is indeed due to the V-groove shape of the QWs.

The spontaneous anisotropy of the V-groove QW grating naturally yields a sensitivity to the polarization of light, especially at normal incidence. When the light is linearly polarized parallel to the $c$ axis, the absorption is zero, whereas for light polarized perpendicular to the $c$ axis, there is a sizable IR absorption. Thus, the question that arises is what the photocurrent polarization contrast would be in a potential device fabricated with these structures. As opposed to a transmission polarizer, where the ratio of the crossed to parallel polarizations can be given as a measure of the quality of the polarizer, here we cannot do the same, since, for the parallel polarization case, the signal of the photodetector would be zero and the ratio would be noninformative or rather unphysical. Thus, it is assumed that the light is incident at normal incidence on the sample with a $\pm 5^{\circ}$ cone of angles.

To compute a figure of merit of this potential photodetector, the integrated absorption intensity in the range $-5^{\circ}<\theta_{\text {ext }}<+5^{\circ}$ is computed from the model. The results are plotted in Fig. 6(a) as a function of $\phi$, normalized to the value when $\phi=0$. It can be observed that the polarization contrast increases with $\phi$ as expected. For sample $\mathrm{B}$, where $\varphi=14^{\circ}$, the contrast would be larger than 80:1 when $\mathbf{E} \perp c$ compared to $\mathbf{E} \| c$. This result can be compared to the published literature. A selectivity ratio of 3:1 was found by Chen et al. using ( $\mathrm{Al}, \mathrm{Ga}) \mathrm{As} / \mathrm{GaAs} \mathrm{QWs}$ that are corrugated by chemical etching [8]. Antoni et al. [6] reported a contrast of $0.6: 1$ also from $(\mathrm{Al}, \mathrm{Ga}) \mathrm{As} / \mathrm{GaAs}$ QWs featuring back-side-etched parallel stripes, whereas Beekman et al. reported a contrast of 2:1 following a similar approach [17]. Therefore, it seems that the approach proposed here could yield a much larger polarization contrast than the GaAs technology with no need for postprocessing of the structures and for nearly collimated light at normal incidence.

Finally, the integrated intensity of the experimental ISB transition as a function of the angle between the light polarization axis and the $c$ axis at normal incidence is plotted in the polar diagram of Fig. 6(b) for sample B $\left(\phi=14^{\circ}\right)$ and compared to the model. The calculation is performed by adding the absorption of the component of the electric field parallel to the $c$ axis to that perpendicular to the $c$ axis. Note that this calculation is also

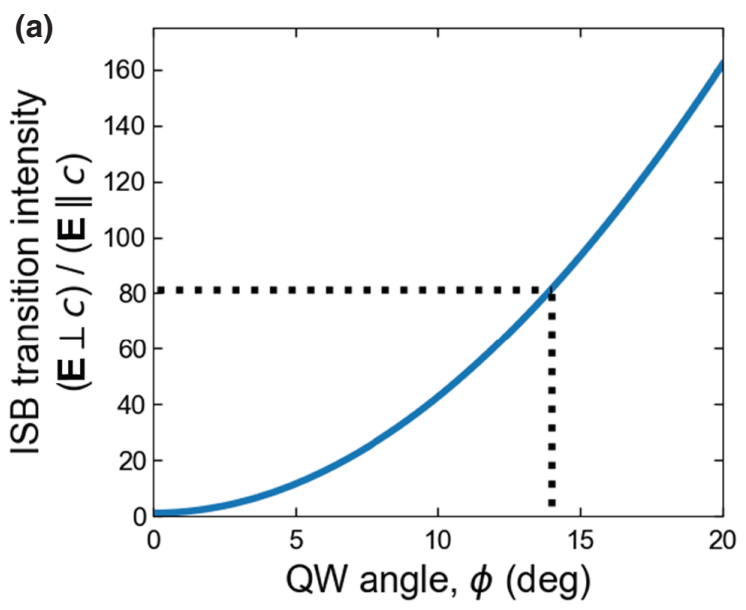

(b) $\mathbf{E} \perp \mathrm{C} \quad 80^{\circ}$

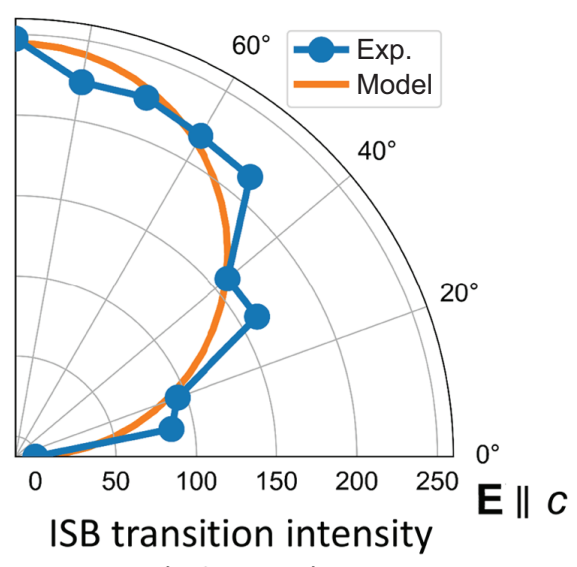

(arb. units)

FIG. 6. (a) Calculated polarization contrast at normal incidence as a function of the $\mathrm{V}$-groove angle $\phi$ for light with a $\pm 5^{\circ}$ cone of angles. The value for $\phi=14^{\circ}$ is indicated with a dotted line. (b) Experimental ISB transition intensity at normal incidence as a function of the polarization with respect to the $c$ axis (blue dots). The orange line is the equivalent calculation from the model.

performed at normal incidence with $\mathrm{a} \pm 5^{\circ}$ cone of angles as assumed previously. The qualitative agreement [note that the proportionality constant $A$ in Eq. (4) is left as a fitting parameter] is excellent between model and experiment, adding further evidence of the validity of the simple geometrical model presented here.

\section{CONCLUSION}

To summarize, we have demonstrated that nonpolar $m$-plane $\mathrm{ZnO} /(\mathrm{Mg}, \mathrm{Zn}) \mathrm{O} \mathrm{MQWs}$ can be used to break the ISB absorption selection rules under normal incidence thanks to their as-grown, anisotropic QW morphology. These MQWs exhibit polarization-selective ISB absorption at normal incidence, with maximum absorption when the electric field of the light is perpendicular to the $c$ axis, 
which is found in-plane. Through IR transmission spectroscopy as a function of the angle of incidence, we have demonstrated that the polarization-sensitive absorption at normal incidence is due to the $\mathrm{V}$-groove grating shape of the QWs. Modeling the ISB absorption coefficient for the geometry of the $\mathrm{V}$-groove MQW grating, we have been able to extract its specific angle with extraordinary accuracy and it has been confirmed by HRTEM image analysis. Finally, we have estimated the polarization contrast for our sample when light is incoming at normal incidence with a $\pm 5^{\circ}$ cone of angles and found a 80:1 polarization sensitivity when $\mathbf{E} \perp c$ vs $\mathbf{E} \| c$.

\section{ACKNOWLEDGMENTS}

Part of the calculations performed here used the Aestimo solver (http://www.aestimosolver.org/). This work was funded by the Spanish Ministry of Economy and Competitiveness (MINECO) through Projects No. TEC201460173-C2-2 and No. TEC2017-85912-C2-1-R and from the European Union's Horizon 2020 Research and Innovation Program under Grant No. 665107 (project ZOTERAC). The authors would like to acknowledge Professor Borge Vinter for his critical reading of the manuscript. J.T.A. holds a Predoctoral Contract from the Universidad Politécnica de Madrid.

[1] G. Günter, A. A. Anappara, J. Hees, A. Sell, G. Biasiol, L. Sorba, S. De Liberato, C. Ciuti, A. Tredicucci, A. Leitenstorfer, and R. Huber, Sub-cycle switch-on of ultrastrong light-matter interaction, Nature 458, 178 (2009).

[2] J. Lee, M. Tymchenko, C. Argyropoulos, P.-Y. Chen, F. Lu, F. Demmerle, G. Boehm, M.-C. Amann, A. Alù, and M. A. Belkin, Giant nonlinear response from plasmonic metasurfaces coupled to intersubband transitions, Nature 511, 65 (2014).

[3] H. Schneider and H. C. Liu, Quantum Well Infrared Photodetectors. Physics and Applications (Springer-Verlag, Berlin Heidelberg, 2007).

[4] F. R. Giorgetta, E. Baumann, M. Graf, Q. Yang, C. Manz, K. Köhler, H. E. Beere, D. A. Ritchie, E. Linfield, A. G. Davies, Y. Fedoryshyn, H. Jäckel, M. Fischer, J. Faist, and D. Hofstetter, Quantum Cascade Detectors, IEEE J. Quantum Electron. 45, 1039 (2009).

[5] J. Scott Tyo, D. L. Goldstein, D. B. Chenault, and J. A. Shaw, Review of passive imaging polarimetry for remote sensing applications, Appl. Opt. 45, 5453 (2009).

[6] T. Antoni, A. Nedelcu, X. Marcadet, H. Facoetti, and V. Berger, High contrast polarization sensitive quantum well infrared photodetectors, Appl. Phys. Lett. 90, 201107 (2007).

[7] A. Berurier and A. Nedelcu, Optimization of light polarization sensitivity in QWIP detectors, Infrared Phys. Technol. 59, 118 (2013).

[8] C. J. Chen, K. K. Choi, L. Rokhinson, W. H. Chang, and D. C. Tsui, Corrugated quantum well infrared photodetectors for polarization detection, Appl. Phys. Lett. 74, 862 (1999).

[9] V. Apalkov, G. Ariyawansa, A. G. Unil Perera, M. Buchanan, Z. R. Wasilewski, and H. C. Liu, Polarization sensitivity of quantum well infrared photodetector coupled to a metallic diffraction grid, IEEE J. Quantum Electron. 46, 877 (2010).

[10] M. Montes Bajo, J. Tamayo-Arriola, M. Hugues, J. M. Ulloa, N. Le Biavan, R. Peretti, F. H. Julien, J. Faist, J.-M. Chauveau, and A. Hierro, Multisubband Plasmons in Doped ZnO Quantum Wells, Phys. Rev. Applied 10, 024005 (2018).

[11] M. Belmoubarik, K. Ohtani, and H. Ohno, Intersubband transitions in $\mathrm{ZnO}$ multiple quantum wells, Appl. Phys. Lett. 92, 191906 (2008).

[12] K. Zhao, G. Chen, B. S. Li, and A. Shen, Mid-infrared intersubband absorptions in $\mathrm{ZnO} / \mathrm{ZnMgO}$ multiple quantum wells, Appl. Phys. Lett. 104, 212104 (2014).

[13] N. Le Biavan, M. Hugues, M. Montes Bajo, J. TamayoArriola, A. Jollivet, D. Lefebvre, Y. Cordier, B. Vinter, F.-H. Julien, A. Hierro, and J.-M. Chauveau, Homoepitaxy of non-polar $\mathrm{ZnO} /(\mathrm{Zn}, \mathrm{Mg}) \mathrm{O}$ multi-quantum wells: From a precise growth control to the observation of intersubband transitions, Appl. Phys. Lett. 111, 231903 (2017).

[14] E. Di Russo, L. Mancini, F. Moyon, S. Moldovan, J. Houard, F. H. Julien, M. Tchernycheva, J.-M. Chauveau, M. Hugues, G. Da Costa, I. Blum, W. Lefebvre, D. Blavette, and L. Rigutti, Three-dimensional atomic-scale investigation of $\mathrm{ZnO}-\mathrm{Mg}_{x} \mathrm{Zn}_{1-x} \mathrm{O} m$-plane heterostructures, Appl. Phys. Lett. 111, 032108 (2017).

[15] A. Delteil, A. Vasanelli, Y. Todorov, C. Feulliet Palma, M. Renaudat St-Jean, G. Beaudoin, I. Sagnes, and C. Sirtori, Charge-Induced Coherence between Intersubband Plasmons in a Quantum Structure, Phys. Rev. Lett. 109, 246808 (2012).

[16] T. Ando, A. B. Fowler, and F. Stern, Electronic properties of two-dimensional systems, Rev. Mod. Phys. 54, 437 (1982).

[17] D. W. Beekman and J. Van Anda, Polarization sensitive QWIP thermal imager, infrared detectors and focal plane arrays VI, Proc. SPIE 4028, 102 (2000). 